\title{
SALUD Y BIENESTAR PSICOLÓGICO: VIOLENCIA INTRAFAMILIAR Y SU INFLUENCIA EN LA AUTOESTIMA DE LOS ESCOLARES. REGIÓN 5 ECUADOR- 2014 HEALTH AND PSYCHOLOGICAL WELL-BEING: INTRAFAMILY VIOLENCE AND ITS INFLUENCE ON THE SELF-ESTEEM OF SCHOOLCHILDREN. REGION 5 ECUADOR- 2014 \\ SAUUDE E BEM-ESTAR PSICOLÓGICO: VIOLÊNCIA INTRAFAMILIAR E SUA INFLUÊNCIA NA AUTOESTIMA DE ESCOLARES. REGIÃO 5 EQUADOR- 2014
}

\section{CARMEN GRACIELA ZAMBRANO VILLALBA ${ }^{1}$}

${ }^{1}$ Universidad Estatal de Milagro, Guayas, Ecuador

RESUMEN

Introducción: la violencia intrafamiliar, es un tipo de violencia relacional que afecta directamente en el desarrollo integral de los escolares. La niñez y adolescencia es una etapa de vulnerabilidad, que sumado a la incidencia de la violencia en el sistema familiar, afecta significativamente en la formación del autoestima, autoconcepto y desarrollo integral de la personalidad. La autoestima es el aprecio, consideración que uno tiene de sí mismo, se forma por la percepción, pensamientos y sentimientos que surgen en el contacto relacional en el sistema familiar, escolar y social. Objetivos: describir la influencia de la violencia intrafamiliar en el autoestima de los escolares de 10 a 14 años, desde el análisis del clima familiar con las subvariables: cohesión, expresividad, conflicto, apoyo, confianza y comunicación. Metodología: se realizó un estudio cuantitativo de tipo transversal, cuyos participantes fueron 13,776 escolares de básica superior de 106 instituciones educativas de la "región 5", Ecuador. Se utilizó la Escala de Clima Familiar de Moos y Moos y el cuestionario de autoestima CARZAM III de Zambrano C., que permitieron obtener información relevante para el estudio por un lapso de 8 meses. Resultados: se determinó que el $88 \%$ presentaron falta de apoyo en las relaciones interpersonales, 98\% falta de comunicación, $62 \%$ de conflicto caracterizado por violencia física y psicológica de pareja, que afectó al desarrollo de la autoestima de los escolares en 41\%. Discusión: la violencia intrafamiliar influyó en el nivel de autoestima de los niños y niñas en la etapa escolar con prevalencia de edad, género y nivel de escolaridad, caracterizado por niveles de desestima medio-bajo y bajo, con comportamientos relacionales conflictivos y violentos.

PALABRAS CLAVE: bienestar psicológico, violencia, autoestima, autoimagen.

ABSTRACT

Introduction: intrafamily violence is a type of relational violence that directly affects the integral development of schoolchildren. Childhood and adolescence are a stage of vulnerability, which, added to the incidence of violence in the family system, significantly affects the formation of self-esteem, self-concept, and integral development of the personality. Self-esteem is the appreciation, the consideration, that one has of himself, it is formed by the perception, thoughts, and feelings that arise in the relational contact in the family, school, and social system. Objectives: describe the influence of domestic violence on the self-esteem of schoolchildren aged 10 to 14 years, from the analysis of the family climate with the subvariables: cohesion, expressiveness, conflict, support, trust, and communication. Methodology: a cross-sectional quantitative study was carried out, whose participants were 13,776 high school students from 106 educational institutions in "region 5", Ecuador. The Moos and Moos Family Environment Scale and the CARZAM III self-esteem questionnaire by Zambrano $C$. were used, which allowed obtaining relevant information for the study for the period of 8 months. Results: it was determined that $88 \%$ presented a lack of support in interpersonal relationships, $98 \%$ lack of communication, $62 \%$ conflict characterized by physical and psychological violence from the partner, which affected the development of self-esteem of schoolchildren in 41\%. Discussion: intrafamily violence influenced the level of self-esteem of boys and girls in the school stage with the prevalence of age, gender, and schooling level, characterized by low-medium and low levels of self-esteem, with conflictive and violent relational behaviors.

KEYWORDS: psychological well-being, violence, self-esteem, self-concept.

RESUMO

Introdução: a violência intrafamiliar é uma forma de violência relacional que afeta diretamente o desenvolvimento integral dos escolares. A infância e a adolescência são uma fase de vulnerabilidade que, somada à incidência de violência no sistema familiar, afeta significativamente a formação da autoestima, do autoconceito e do desenvolvimento integral da personalidade. A autoestima é a valorização, consideração que se tem de si, é formada pela percepção, pensamentos e sentimentos que surgem no contato relacional na família, na escola e no sistema social. Objetivos: descrever a influência da violência doméstica na autoestima de escolares de 10 a 14 anos, a partir da análise do clima familiar com as subvariáveis: coesão, expressividade, conflito, apoio, confiança e comunicação. Metodologia: foi realizado um estudo transversal quantitativo, cujos participantes foram 13.776 alunos do ensino médio de 106 instituições de ensino da "região 5" do Equador. Utilizou-se a Escala de Clima Familiar de Moos e Moos e 0 questionário de autoestima CARZAM III de Zambrano C., que permitiram obter informações relevantes para 0 estudo por um período de 8 meses. Resultados: constatou-se que $88 \%$ apresentavam falta de apoio nas relações interpessoais, $98 \%$ falta de comunicação, $62 \%$ conflito caracterizado por violência física e psicológica do companheiro, que afetou o desenvolvimento da autoestima dos escolares em $41 \%$. Discussão: a violência intrafamiliar influenciou o nível de autoestima de meninos e meninas em fase escolar com prevalência de idade, gênero e escolaridade, caracterizada por níveis médio-baixo e baixo de autoestima, com comportamentos relacionais conflituosos e violentos.

PALAVRAS-CHAVE: bem-estar psicológico, violencia, autoimagen. 
INTRODUCCIÓN

La convivencia familiar y el bienestar emocional, psicológico y social es importante en el desarrollo de la personalidad de los seres humanos. ${ }^{22} \mathrm{La}$ seguridad, necesidad de aprobación, autoestima, autoconcepto, autopercepción y autovaloración están íntimamente relacionados al desarrollo psico-efectivo. La Organización Mundial de la Salud. ${ }^{1}$ definió a la salud mental como "El estado de bienestar físico, mental, social y no solo ausencia de enfermedad" que permite tener una visión amplia de la salud y bienestar psicológico. La American Psychological association2 aborda el tema de salud y bienestar como una conexión armónica entre el cerebro y el cuerpo que, frente a factores externos o internos, puede causar desequilibrio, entre ellas el distres, la violencia y situación económica. Whittingham ${ }^{3}$ determinó la importancia de la salud mental en todas las dimensiones de personalidad, a la que consideró como el estado de bienestar físico, emocional, psicológico, social y espiritual que le permiten participar en armonía con el ambiente en su contexto. La presencia de salud mental promueve el bienestar psicológico de las personas, permite el interaprendizaje, tomar decisiones y obtener salud y bienestar con un estilo de vida saludable.

Desde esta perspectiva, el bienestar es considerado como el estado armónico apropiado de las diversas dimensiones de las personas desde su desarrollo, generando buen nivel de autoestima para enfrentar las exigencias de la vida cotidiana, generando un bienestar emocional, escolar, familiar y social con relaciones interpersonales efectivas y asertivas. La adolescencia tiene un proceso de profundos cambios biopsicosociales, ${ }^{24}$ que generan crisis y vulnerabilidad emocional, predisponen a dar origen a trastornos psicológicos como trastornos disociales y de humor, distimias, depresiones, ideación suicida, delincuencia, consumo de drogas y otras alteraciones conductuales.

El adolescente promedio manifiesta aburrimiento, pérdida de interés, desmotivación y/o tendencias a actuaciones antisociales o apatía (poco interés) ante situaciones que de lo contrario serían atractivas. Es muy factible encontrar comportamientos más bien agresivos, descalificadores y desafiantes. La violencia familiar es un tipo de violencia doméstica ${ }^{20}$ con un patrón de conducta en desigualdad de poder caracterizada por violencia física y psicológica entre otras que influye en los miembros más vulnerables del hogar, como son los niños en general que se encuentran en la etapa escolar. ${ }^{14}$ La naturaleza del problema y su importancia radica en los procesos de ajuste psicoemocionales, en las relaciones comunicacionales y afectivas interpersonales e intrapersonales en el sistema familiar, escolar y social que favorece o no al desarrollo de la autoestima.

El objetivo de este trabajo es analizar la influencia de la violencia intrafamiliar sobre la autestima de los niños y niñas de 10 a 14 años en el ambiente socioeducativo, identificar el clima familiar y violencia intrafamiliar, establecer los diferentes niveles de autoestima y analizar como incide en el comportamiento social del grupo etario señalado, que sufren violencia intrafamiliar. La hipótesis planteada es: la violencia del sistema familiar afecta la autoestima de los escolares

\section{METODOLOGÍA}

El diseño de investigación es transversal. La población del estudio está compuesta por niños provenientes de las provincias de la "Región 5" del Ecuador que asciende a 13,776 escolares de básica superior de 106 instituciones educativas, de edades comprendidas entre 10 a 14 años de ambos sexos.

Para medir la variable violencia intrafamiliar se utilizó la escala de clima familiar de Moos y Moos $^{21}$ de 30 items, con modificaciones de acuerdo al contexto socio cultural en los indicadores de conflicto/ violencia, la misma que permitió codificar la Cohesión, expresividad y conflicto en las relaciones interpersonales. El ítem "mis padres nunca están y cuando están pelean frecuentemente permite evidenciar la variable violencia intrafamiliar. Los índices de fiabilidad en otros estudios han sido muy satisfactorios, al igual que la validez de los ítems que permiten medir las variables descritas en el clima familiar.

Para medir la variable autoestima se utilizó el Test de autoestima CARZAM III de Zambrano ${ }^{22}$ validados por expertos y aplicado en otras poblaciones de niños y adolescentes de diferentes niveles educativos para detectar los niveles de estima y desestima en casos con trastornos disociales, trastornos del humor, variabilidad en el auto concepto, autoconocimiento y autoestima. Para la recolección de la información en las instituciones educativas se solicitó por escrito la autorización de la dirección Zonal de la región 5 respaldado por el convenio interinstitucional entre la Universidad Estatal de Milagro UNEMI y el Ministerio de Educación. Se efectuó las visitas a las instituciones educativas 
de cantones de cada provincia, se procedió a la recolección de la información pertinente por un lapso de 8 meses y se realizó la aplicación de los cuestionarios previamente validados. La aplicación de los cuestionarios se realizó en horarios de mañana y tarde en forma grupal dentro del aula en un tiempo aproximado de 30 minutos.

Luego de aplicar los cuestionarios, se realizó el análisis estadístico informático en el programa SPSS de los datos demográficos de edad, género y escolaridad de la muestra. Se utilizó la correlación de Pearson para análisis la influencia entre variables, clima familiar y autoestima con sus subvariables de cohesión, expresividad, conflicto, con frecuencia de edad, genero, escolaridad y niveles de autoestima en los escolares.

RESULTADOS

De la población muestral expresada en el cuadro 1 calculado a partir de los datos agrupados, se desprende la edad promedio de 12 años con un $37,3 \%$,con una variabilidad de, 014 . La asimetría estuvo por encima de la mediana y la curtosis por debajo de la misma, tal como se aprecia en el cuadro 1 . Se presenta la frecuencia estadística de género que se obtiene como resultado del análisis descriptivo de los datos, en donde 6727 son mujeres que corresponde al 48\% y 7049 son hombres.. Dentro del análisis del clima intrafamiliar se evidenció la presencia de la subvariable "Cohe- sión” en un 88\% que representó la relación entre los miembros de la familia.

Otro de los factores que se analizó fue la subvariable expresividad en las relaciones intrafamiliares determinando que el 98\% tiene buena comunicación y el mismo porcentaje se presentó en la falta de comunicación entre los subsistemas y sus relaciones interpersonales en el ámbito familiar. Por otro lado, la subvariable "Conflicto en las relaciones interpersonales dentro de la familia" se encontró que el $62 \%$ estuvo caracterizado por violencia física en un $84 \%$ entre el subsistema de pareja y $78 \%$ de violencia psicológica en relación entre los miembros del subsistema familiar padre-hijo y madre-hijo dentro del sistema familiar. Se realizó un análisis correlacional entre las subvariables, cohesión, expresividad y conflicto como parte del clima intrafamiliar de los escolares, los mismos que se caracterizaron por relaciones interpersonales conflictivas dentro del sistema familiar, obteniendo porcentajes e indicadores descriptivos de los valores obtenidos expresados y evidenciados en el cuadro 1 del estudio.

Por último, se analizó la variable autoestima, resultados que evidenció dos niveles de desestima, como fue el nivel medio-bajo y el nivel bajo, ambos niveles suman el $41 \%$ de la muestra que tienen promedios medios bajos y bajo nivel de autoestima, mientras que el $25 \%$ de los escolares, se encontró en un nivel medio y por otro lado el

TABLA 1. DESCRIPCIÓN DE LA POBLACIÓN DE ESTUDIO

\begin{tabular}{|c|c|c|c|c|c|c|c|c|}
\hline \multicolumn{3}{|c|}{ Frecuencia de edad } & \multicolumn{3}{|c|}{ Frecuencia de género } & \multicolumn{3}{|l|}{ Clima familiar } \\
\hline Edad & Frecuencia & Porcentaje & Género & Frecuencia & Porcentaje & subvariables & Descripción & Porcentaje \\
\hline 10 & 2271 & $16,4, \%$ & Mujer & 6727 & $48,0 \%$ & \multirow{4}{*}{ Cohesión } & Apoyo & \\
\hline 11 & 3595 & $25,9, \%$ & Hombre & 7049 & $52,0 \%$ & & Falta de apoyo & $88 \%$ \\
\hline 12 & 5182 & $37,3, \%$ & Total & 13776 & $100,0 \%$ & & Confianza & \\
\hline 13 & 1843 & $13,3, \%$ & \multicolumn{3}{|c|}{ Violencia intrafamiliar } & & Desconfianza & \\
\hline \multirow[t]{3}{*}{14} & 885 & $6,4 \%$ & Tipos & Verdadero & Falso & Expresibidad & Comunicación & $98 \%$ \\
\hline & & & Físico & $16 \%$ & $84 \%$ & & F/comunicación & \\
\hline & & & Psicológico & $22 \%$ & $78 \%$ & Conflicto & Violencia/pareja & $62 \%$ \\
\hline Total & 13.776 & $100.0 \%$ & & & & & P/hijo-M/hijo & \\
\hline
\end{tabular}

$30 \%$ de la muestra tuvo alto nivel de autoestima. Sumado a los determinantes socioafectivos del sistema familiar, descritos en la tabla 1 se puede determinar la descripción de los niveles de autoestima de la población de estudio.
La edad representativa que sufren violencia intrafamiliar y afectó al autoestima de los participantes estuvo entre 12 a 13 años con $23 \%$, mientras que el $21 \%$ correspondió al género masculino, los mismos que tuvieron problemas de autoestima caracterizados por disminución de la motivación 


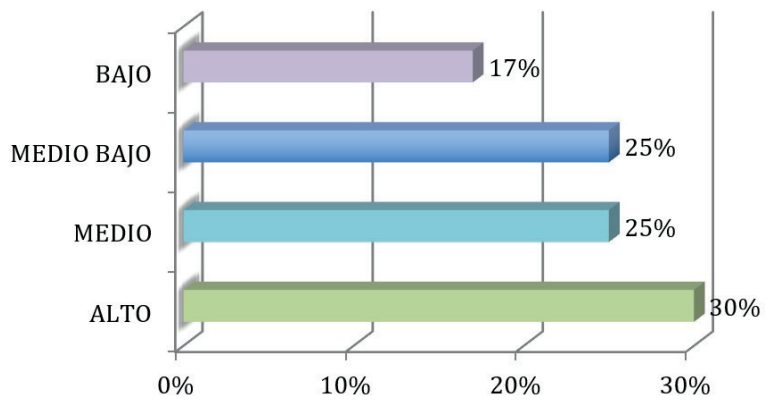

Figura. Niveles de autoestima.

y desconfianza entre los miembros del sistema familiar; mientras que $15 \%$ del total de mujeres presentaron niveles de desestima caracterizado por presencia de conflictos, bajo concepto de sí mismo y por ende de su autoestima. El 35\% los escolares que sufren de problemas de autoestima se encontró en octavo de básica, el $12 \%$ en noveno; y el $5 \%$ en décimo de básica superior de acuerdo a los niveles de educación.

Los resultados más importantes del estudio estuvieron en relación al ambiente conflictivo caracterizado por violencia física y psicológica que repercutió en la autoestima en un $41 \%$ carac- $^{-}$ terizado por niveles de desestima medio-bajo y bajo en el grupo vulnerable del sistema familiar que incidieron en el comportamiento relacional de los escolares y en la autoestima. Dentro del clima familiar, la variable conflicto se presentó desde el subsistema de pareja hasta el subsistema de los hijos, verificado en las relaciones interpersonales en el ambiente familiar y escolar que se caracterizó por mucho esfuerzo para mantener un buen clima familiar, falta de apoyo en las relaciones interpersonales, entre padre-hijo/a y madre hijo/a, que trascienden a vínculos afectivos e interpersonales entre compañeros y maestros.

DISCUSIÓN

En un estudio de la comunicación familiar y valoración parental en conducta violenta en 665 adolescentes en edades comprendidas de 12 a 16 años, se analizó la influencia familiar en la autoestima del adolescente y su actitud frente a la autoridad escolar determinando que ésta incide significativamente en la conducta violenta del escolar ${ }^{16}$ representado por una actitud afectiva disminuida hacia sí mismo, coincidiendo con los resultados obtenidos en el estudio, en donde la violencia física y psicológica contribuyó a la presencia de bajo nivel de autoestima.
En la evaluación del autoestima17 en 866 escolares, determina que la violencia está íntimamente relacionada al desarrollo afectivo del escolar. Los estudios de la autoestima en diferentes investigaciones están relacionados con el contexto educativo18 con el propósito de comprender los procesos de la conducta en las relaciones interpersonales y en el desarrollo académico y socioeducativo. En cuanto a la correlación de edad y autoestima se evidenció que entre menos es la edad y exista presencia de violencia familiar, mayor es la probabilidad de que se encuentre disminuida la autoestima de los escolares. Referente al género se pudo interpretar que existieron tendencias de sufrir más problemas en el desarrollo de la autoestima en hombres que en mujeres, aunque en mujeres existieron más rasgos evidentes de conducta con depresión, distimias y prácticas de cutting.

En otra investigación referente a la autoestima de los escolares ${ }^{4}$ concluyen que está basada en la dimensión afectiva, considerando el nivel madurativo y experimental. La violencia influye en el nivel de autoestima porque la percepción violenta del contexto genera pensamientos negativos de sí mismo y de los demás. Aumenta la preocupación que impide la concentración, genera inseguridad y baja autoestima.

En la investigación referente al autoconcepto y autoestima en el aprendizaje de los escolares, González J, Núñez C. Glez S. García M..$^{5}$ determinó que la personalidad está organizada por una estructura funcional que va desde lo sensorial, motor, cognitivo, afectivo, estilo y valores: la interacción de los mismos da como resultado la conducta. Los procesos cognitivos y afectivos son los que influyen directamente en la personalidad expresada en tres dimensiones como es la visión del mundo, estilo de vida, autoimagen, autoconcepto y autoestima.

Maslow ${ }^{18}$ en su estudio de las jerarquías de las necesidades humanas describe a la necesidad de autoestima como parte del ámbito de la personalidad que se caracteriza por amor propio, confianza, aprecio hacia sí mismo. Asociada a la autoimagen que se tiene de uno mismo, las mismas que dependen de nuestras interacciones y experiencias vitales que constituyen nuestra personalidad. Goleman en su estudio de inteligencia emocional ${ }^{6}$ manifiesta que las emociones nos movilizan a la acción a través de una serie de procesos cognitivos en 
relación permanente entre el pensamiento y los sentimientos, dotado de habilidades como el autocontrol o dominio de uno mismo y de las emociones que permiten alcanzar el bienestar personal a partir de la autoestima.

Gardner $^{7}$ desde sus bases neurocientíficas, explica que la autoestima es una expresión de la inteligencia intrapersonal que permite la reflexividad, pensamiento asertivo, entenderse así mismo, reconocimiento de fortalezas y debilidades. La autoestima es la valoración positiva o negativa de uno mismo en función a procesos metacognitivos, determinado por pensamientos, sentimientos, experiencias, relacionada con la autoimagen o concepto de uno mismo y de la autoaceptación de las cualidades y defectos. ${ }^{8}$

Cuando las condiciones personales y familiares no tienen bienestar, la autoestima baja y ésta repercute en el comportamiento, relaciones interpersonales y estado de ánimo; afecta también en el aprendizaje de los escolares. ${ }^{9}$ La presencia de violencia intrafamiliar produce deterioro de la salud mental y del bienestar personal y social de los escolares, especialmente si hay exposición de violencia éstos tienden a presentar disminución de la autoestima, autoconcepto e inestabilidad en la personalidad.

Horntein,,$^{10}$ desde una fundamentación neurocientífica, manifiesta que la autoestima se forma como un proceso de cambio y trasformación desde la niñez hasta la formación completa de la personalidad, la misma que debe ser comprendida desde las condiciones personales y sociales en que los niños se desarrollan. Cuando existen condiciones intrafamiliares como la violencia ${ }^{19}$ se expone al niño o niña a generar problemas en la autoestima determinando niveles bajos, medios o altos de autoestima caracterizados por variaciones en la predisposición del escolar al aprendizaje, en sus tomas de decisiones, en su autoconfianza mostrándose inseguros, muy sensibles y con pocas expectativas para resolver problemas. Boyce en su estudio de la personalidad ${ }^{11}$ señala que ésta influye significativamente en la vida personal y familiar de las personas con implicaciones a los miembros de la familia. En otro estudio de la violencia intrafamiliar y las relaciones interpersonales de los escolares ${ }^{12}$ se analizó las variables clima familiar y clima escolar obteniendo como resultado el $77 \%$ de conflictos de parejas que afectan directamente en sus niveles de confianza, apoyo en las relaciones intrafamiliares y el $72 \%$ en la cohesión, expresividad y conflicto en las relaciones interpersonales en el ambiente escolar. Según el informe mundial de la prevención de la violencia en el mundo $\mathrm{OMS}^{13}$ la violencia afecta a los grupos vulnerables de la sociedad, esta disminuye la salud y bienestar personal, familiar y social. Estevez, E. Martinez, b. Musitu, G. en sus estudios de Intervención psicosocial, ${ }^{15}$ analizan la autoestima en adolescentes agresores y víctimas en el ambiente escolar, presentan en general, las puntuaciones más bajas de las dimensiones de autoestima.

CONCLUSIÓN

En este estudio se realizó una descripción de la influencia de la violencia intrafamiliar en la autoestima que afecta al desarrollo de la salud y bienestar de la personalidad y en la convivencia escolar de los participantes. En efecto los resultados establecieron que existe prevalencia de violencia de pareja cuyas implicaciones se evidencian en el comportamiento agresivo de los estudiantes en el ambiente escolar.

Se estableció el nivel de rechazo y aceptación en las relaciones familiares y escolares siendo la comunicación la más afectada y este a su vezes uno de los factores que inciden negativamente en las relacionesinterpersonales. Alcontrastarlos resultados obtenidos se obtuvieron niveles altos de cohesión expresividad y conflicto en el comportamiento relacional, además, sedeterminólostiposdeviolencia, en las relaciones interpersonales y comunicacionales dentro del sistema familiar.Cabe destacar que la importancia y relevancia de este estudio son los hallazgos encontrados que se convierten en aportes significativos para comprender el fenómeno de la violencia intrafamiliar en el contexto de la región 5 del Ecuador, y aporta a la promoción de la salud mental de la población vulnerable de la sociedad. Por último los resultados encontrados permiten comprender el comportamiento socio familiar en relación a la funcionalidad dela pareja y en relación de padres a hijos y de hijos a padres, para entender las relaciones interpersonales en el sistema escolar.

\section{RECOMENDACIONES}

De acuerdo a los resultados obtenidos es necesario incluir en el proceso de intervención psicológica a la familia, espacios de comunicación que permitan disminuir la distancia emocional, el alto nivel de cohesión relacional, expresividad y conflicto en los subsistemas y favorecer el clima socio familiar y por ende disminuir la presencia de factores que lesionen la autoestima. 
Es evidente que los conflictos de pareja ocasionan el deterioro del clima intrafamiliar y este a su vez influye decisivamente en el autoestima y violencia relacional escolar en la dinámica entre pares de los niños expuestos a violencia, por tal motivo es preciso y pertinente promover una relación de pareja fundamentado en afectos positivos, acuerdo, respeto, confianza, motivación y toma dedecisiones con espacios abiertos para la comunicación y asertividad en las relaciones interpersonales y en la dinámica intrafamiliar,

Almejorar las relaciones de pareja y generarespacios que favorezcan el bienestar de los miembros del hogar con vínculos afectivos interpersonales y comunicacionales positivos, los niños y niñas en los espacios escolares podrán fomentar relaciones positivas entre pares y favorecer una convivencia armónica para el desarrollo psicológico, en el aspecto emocional, autoestima, autoconcepto que caracteriza a una personalidad sana.

REFERENCIAS BIBLIOGRÁFICAS

1. OMS/OPS. Informe de salud en el mundo. Ginebra. América Latina: Organización panamericana de la salud; 2014.

2. Rodríguez M. Psicología de la salud y psicología clínica. American Psychological Association. 1998; ISNN 0214-7823.

3. Whithinghan, C. Amind that Found Itdself. Estados Unidos;1908.

4. De Tejada, L. Evaluación de la autoestima en un grupo de escolares. Revista de psicología. 2010; Vol.16. ํㅡㄴ 1, pp 95-103.

5. González-Pienda, J. Núñez Pérez, C., Glez.Pumariega, S y García García, M. Psicothema 1997. Vol. 9, no 2, pp. 271-289 ISSN 0214 - 9915.

6. Goleman, D. Inteligencia emocional. $4^{\circ}$ edición. Barcelona, Kairos. 1996; ISBN 97884-7245-371-5.

7. Gardner, H. Inteligencias múltiples. La teoría en la practica $1^{\circ}$ edición. Barcelona Paidos. 2011; ISBN 978-84-493-2594-6.

8. Pérez, C. Bonenfoy, C. Cabrera,A. Peine, S. Muñoz,C. Baquedano,M. Jiménez,J. Análisis, desde la psicología positiva de la salud mental en alumnos universitarios del primer año de Concepción de Chile Avances de la Psicología Latinoamericana Bogotá Colombia. vol. 29 pp149-160

9. Morales, M. González, A. Resilencia, Autoestima, Bienestar psicológico y capacidades intelectuales en estudiantes de cuarto medio de buen rendimiento. 2014; Vol XI. Pp 215-228
10. Horntein, L. Autoestima eidentidad, narcisismo y valores sociales. Buenos Aires Argentina 2011; pp 224.

11. Boyce, C. Personality change follinwing unemployment. Revista investigación y ciencia. 2015

12. Zambrano,C. Violencia intrafamiliar y relaciones interpersonales en los escolares. 2015.

13. ONU. Informe mundial de la violencia y salud. Organización mundial de la salud: Psicothema 2012; univiedo.es.

14. Baldry, A. Child Abuse \& Neglect: Bullying in schools and exposure to domestic violence. Science Direct, 2003; 27, 713-732.

15. Estevez, E. Martínez, b. Musitu, G. Estudios de Intervención psicosocial. 2006; vol 15 pag. 223-232).

16. Cava, M. Musitu, G. Murgui, S. Familia y violencia escolar: El roll mediador de la autoestima y la actitud hacia la autoridad institucional. Psicothema, 2006;18, 03 - pp 367-373.

17. Maslow, A. Una visión humanista para la empresa de hoy. Barcelona. Ed. Paidós Ibérica 2005; ISBN 84-493-1698-7.

18. Gámez, M. Violencia filio parental y su asociación con la exposición a la violencia marital y la agresión de padres a hijos. Psicothema, 2012; 24, 277-283.

19. Evan, S. y Davies, C. Dilillo, D. Exposure to Domestic Violence: A Meta-Analysis of Child and Adolescent Outcomes Published in Aggression and violent behavior. ScieneDirect, 2008; 13, 1359-1789.

20. Fuensanta, C. Violencia intrafamiliar, $\mathrm{Bu}^{-}$ llying. Revista electrónica de investigación Psicoeducativa, Murcia España. 2006; 4, 333-352.

21. Moos, R. y Moos, B. Family Environment Scale Manual: Development, Applications, Research - Third Edition. 1994; Palo Alto, CA: Consulting Psychologist Press.

22. Zambrano, C. Test de autoestima CARZAM III. 2011.

23. Dongil, E. Desarrollo personal y bienestar: Sociedad española para el estudio de la ansiedad y el stress. 2014; 1-17.

24. Villa, M. y Sirvent, C. Desordenes afectivos, crisis de identidad e ideación suicida en adolescentes. International Journal of Psychology and Psychological Therapy 2011; 11, 1, pp. 33-56. 